\title{
"Toy" Dynamo to Describe the Long-term Solar Activity Cycles
}

D. Volobuev

Published online: 21 August 2007

(C) Springer Science+Business Media B.V. 2007

\section{Erratum to: Solar Physics}

DOI: 10.1007/s11207-006-0154-x

Equations (1) and (3) lack a minus sign in front of the $\mu$. This erratum does not effect the calculations or conclusions of the paper.

The online version of the original article can be found at http://dx.doi.org/10.1007/s11207-006-0154-x.

D. Volobuev $(\bowtie)$

Pulkovo Observatory, St.-Petersburg, Russia

e-mail: dmitry.volobuev@mail.ru 